\title{
Ancillary effects of surfactants on filtration of low molecular weight contaminants through cellulose nitrate membrane filters
}

\author{
Aybike N. Olcay ${ }^{\mathrm{a}}$, Mehmet Polat ${ }^{\mathrm{a}}$, Hurriyet Polat ${ }^{\mathrm{b}, *}$ \\ a Izmir Institute of Technology, Department of Chemical Engineering, Izmir, Turkey \\ ${ }^{\mathrm{b}}$ Izmir Institute of Technology, Department of Chemistry, Izmir, Turkey
}

\section{H I G H L I G H T S}

- Membrane filtration is commonly employed for removal of LMWCs.

- Surfactant molecules are invariably present in such waste waters.

- MB removal with a cellulosic membrane was tested in the presence of surfactants.

- Membrane interactions with contaminant and surfactant influence removal strongly.

\section{A R T I C L E I N F O}

\section{Article history:}

Received 8 October 2015

Received in revised form

25 December 2015

Accepted 29 December 2015

Available online 4 January 2016

\section{Keywords:}

Filtration

Ultrafiltration

Surfactant(s)

Micelle(s)

Membrane(s)

\section{G R A P H I C A L A B S T R A C T}

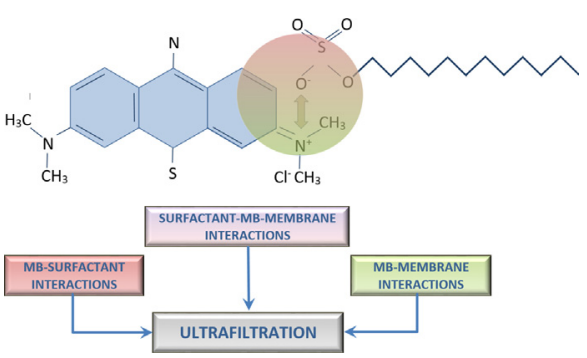

\begin{abstract}
A B S T R A C T
Removal of contaminants with low molecular weight ( $<800$ Dalton) requires the use of advanced separation techniques such as ultrafiltration (UF) or micellar enhanced ultrafiltration (MEUF). However, surface active agents invariably co-exist in waste waters along with these contaminants or they may be added intentionally as part of the separation process as in the case of MEUF. Though it is quite likely that both the filter medium and the contaminants would interact with the surfactant molecules or their micelles, there is not sufficient emphasis in the literature on the concomitant aspects of such interactions.

The ancillary effects created by anionic (sodium dodecyl sulfate, SDS), cationic (hexadecyltrimethyl ammonium bromide, CTAB) and non-ionic (ethoxylated octylphenol, TX-100) surfactants on the mechanism and efficiency of the filtration process were investigated in this study. Methylene blue (MB) and cellulose nitrate membrane (CNM) filters were employed as model retentate and the separation medium. A combination of surface tension, contact angle and charge measurements demonstrated that the addition of surfactants had a remarkable effect on the filtration outcome. The effect depended on both the type and concentration of the surfactant and was manifested mainly through the creation of MB-surfactant entities which acted differently than the MB alone; but more importantly, through the interactions of the surfactant molecules/micelles and the MB-surfactant pairs with the separation membrane.
\end{abstract}

(c) 2015 Elsevier B.V. All rights reserved.

\section{Introduction}

Low molecular weight contaminants (LMWCs) with molecular weights less than 800 Dalton originate from metal-plating,

\footnotetext{
* Corresponding author. Fax: +90 2327507509.

E-mail address: hurriyetpolat@iyte.edu.tr (H. Polat).
}

textile, circuit-board manufacturing, photo-processing, petroleum and mining industries and pose a serious environmental concern (Table 1) [1-7].

The problems with LMWCs are compounded due to removal problems with classical techniques such as chemical oxidation, coagulation, biodegradation, adsorption and extraction owing to smaller molecular size, high solubility and higher dilution rates. The membrane separation is one of the effective methods for removal 

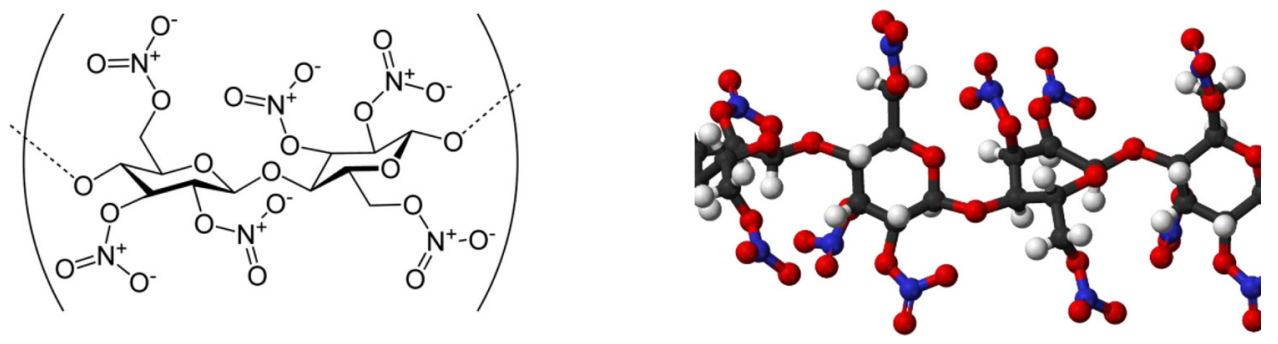

Fig. 1. The cellulose nitrate membrane (CNM) structure.

Table 1

Examples of LMWCs.

\begin{tabular}{ll}
\hline LMWCs & Molecular weight (D) \\
\hline $\begin{array}{l}\text { Polycyclic (2 or 3 fused rings) } \\
\text { aromatic hydrocarbons }\end{array}$ & $152-178$ \\
$\quad$ (PAHs) & \\
Acid anhydrides & $100-218$ \\
Methylene blue (MB) & 374 \\
Cyclonite & 222 \\
Atrazine & 216 \\
2,4-Dichlorophenol & 163 \\
Eosin dye & 692
\end{tabular}

of such contaminants from the wastewater. Ultrafiltration (UF) and micellar enhanced ultrafiltration (MEUF) which fall in this category have been studied extensively to improve both the selectivity and the flux in the removal of these substances [7-17].

Surface active agents which are invariably co-present with the LMWCs in contaminated waters, or added intentionally as part of the ultrafiltration process, may interfere with separation. Though surfactant-contaminant interactions have been addressed to a degree in the literature [7-9], there is not sufficient emphasis on the interaction of the surfactant entities with the membrane material. Moreover, the possible interaction of the membrane with the contaminant itself and with the contaminant-surfactant complexes further compounds the problem. Therefore, a systematic characterization of the system with respect to the magnitude and effect of these interactions is extremely important for properly interpreting both the mechanism and success of separation in such advanced removal technologies.

This work quantitatively demonstrates that interactions of various anionic, cationic and non-ionic surface active agents with a model contaminant, Methylene blue (MB), and with the separation medium, cellulose nitrate membrane, (CNM).

\section{Experimental}

\subsection{Materials}

Selected anionic (sodium dodecyl sulfate, SDS), cationic (hexadecyl trimethylammonium bromide, CTAB) and non-ionic (ethoxyleted octylphenol, TX-100) surface active agents and a model contaminant (methylene blue, $\mathrm{MB}$ ) were employed in the experimental studies (Table 2). All chemicals were reagent grade and obtained from Sigma-Aldrich, United States.

The choice of the separation medium was negatively charged cellulose nitrate membrane (CNM) filters. They were used in two distinct pore sizes of 0.2 and $0.45 \mu \mathrm{m}$ and were supplied by Sartorius Stedim Biotech, Germany. Cellulose nitrate (nitrocellulose) is produced by treating cellulose with nitric acid. Each glucose unit in the cellulose polymer is esterified with three nitrate groups, and these nitrate groups are responsible for both the negative charge of nitrocellulose at neutral $\mathrm{pH}$ and the unusual flammability of dry nitrocellulose (Fig. 1). The negative charge on the membrane sug-

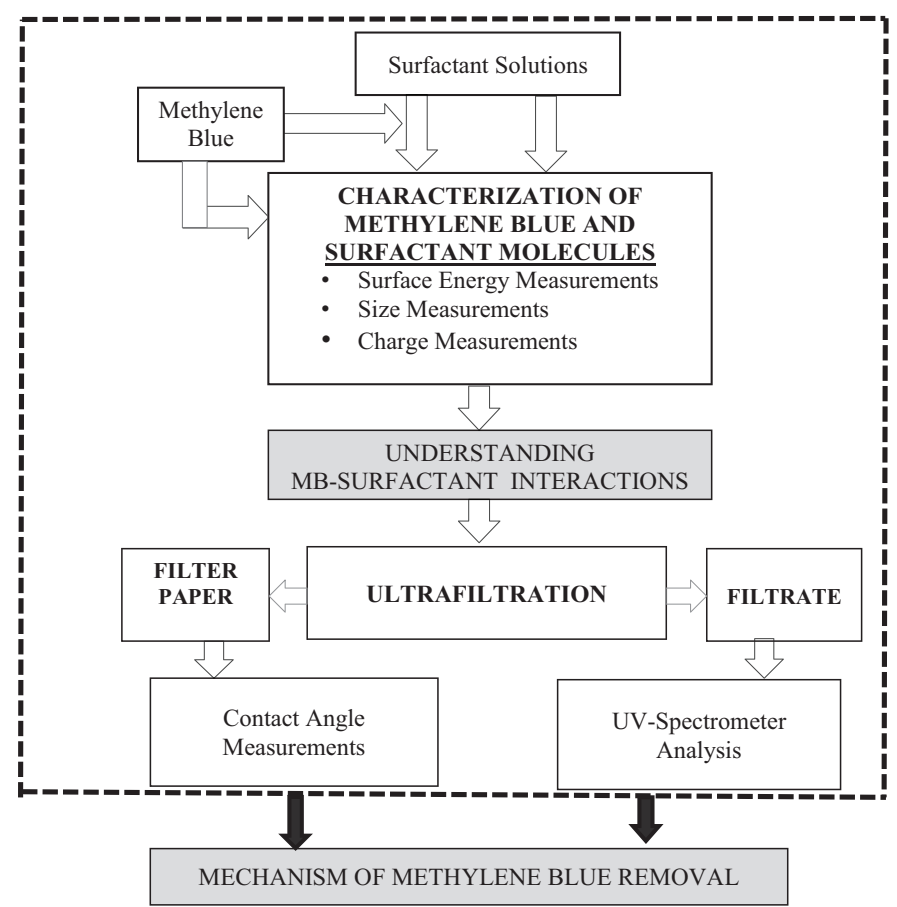

Fig. 2. The methodology employed in this study.

gests that electrostatic interaction may play a significant role in the capture of the contaminants, though it has been stated that hydrophobic interactions also play a role in the capture of proteins of these membranes [18].

\subsection{Methods}

The general methodology followed in this work is presented schematically in (Fig. 2). Surface energy measurements were conducted with a Digital Tensiometer (Kruss K10ST) employing the Du-Noüy Ring method using solutions of SDS, TX-100 and CTAB at concentrations from $10^{-2}$ to $10^{-6} \mathrm{M}$. Zeta potential measurements were carried out with SDS, CTAB and TX-100 solutions in the presence of $10^{-5} \mathrm{M}(\sim 2 \mathrm{ppm}) \mathrm{MB}$ by Laser Doppler Velocimetry (LDV) (Malvern Zeta Sizer Nano ZS). Particle size distribution (PSD) measurements were carried out with the solutions of the same surfactants using Dynamic Light Scattering (DLS) mode of the Zeta Sizer; however, only TX-100 solutions at $10^{-2} \mathrm{M}$ resulted in meaningful PSDs. UV/Vis spectroscopy (PerkinElmer Lambda 45) was used to determine MB concentrations in solutions without and with surfactants of varying strength. Ultrafiltration experiments with the MB-surfactant solutions were carried out at surfactant concentrations from $10^{-2}$ to $10^{-6} \mathrm{M}$ employing the CNM filters with pore sizes of 0.2 and $0.45 \mu \mathrm{m}$. 
Table 2

Some selected properties of the surfactants and the LMW contaminant used.

\begin{tabular}{|c|c|c|c|}
\hline The compound name & MW (Dalton) & Chemical structure & Explanation \\
\hline Sodium dodecyl sulfate (SDS) & 288 & & Anionic surfactant \\
\hline Hexadecyl trimethyl-ammonium bromide (CTAB) & 364 & & Cationic surfactant \\
\hline Ethoxylated octyl phenol (TX-100) C14H22O $(\mathrm{C} 2 \mathrm{H} 4 \mathrm{O})_{n}(n=9-10)$ & 628 & & Nonionic surfactant \\
\hline Methylene blue (MB) & 320 & & Organic contaminant \\
\hline
\end{tabular}

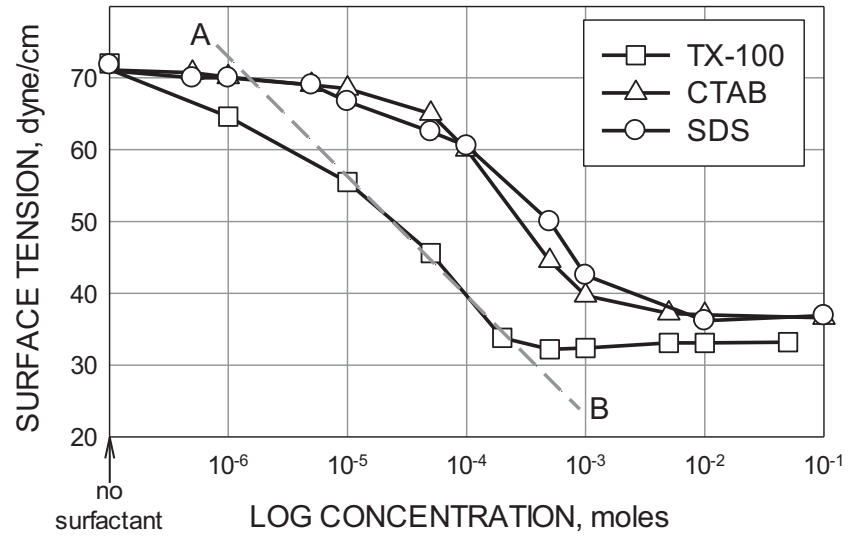

Fig. 3. Surface tension results of SDS, CTAB and TX-100.

\section{Results and discussion}

\subsection{Morphology of surfactant molecules in the absence and presence of $M B$}

Surface tension measurements were performed to determine the association behavior of the surfactant molecules in aqueous surfactant solutions in the absence of the MB and the results are presented in (Fig. 3). The figure shows that the surface tension behavior of the surfactants could roughly be divided into two concentration regions: Region I where the surface tension decreases steadily with increasing concentration until a critical concentration (namely the Critical Micelle Concentration or CMC) is reached, consists principally of monomers. In Region II where the surface tension remains constant with changes in concentration, the surfactant molecules are in the form of micelles. The CMC values determined from the figure are $8 \times 10^{-3} \mathrm{M}$ for SDS, $9 \times 10^{-3} \mathrm{M}$ for $\mathrm{CTAB}$ and $3 \times 10^{-4} \mathrm{M}$ for TX-100. These values agree very well with those reported in literature [19-21]. The part of the surface tension-concentration plot where the surface tension shows a linear decrease with logarithmic concentration (shown by the dashed line A-B) can be used to determine the adsorption density of the surfactant molecules at the air-water interface. According to the Gibbs adsorption equation:

$\Gamma=-\frac{1}{\mathrm{RT}}\left(\frac{\mathrm{d} \gamma}{\mathrm{d} \ln C}\right)$

here $\Gamma$ is the surface excess concentration of the surfactant molecules at the air water interface $\left(\mathrm{mol} / \mathrm{m}^{2}\right), C$ is the bulk concentration $\left(\mathrm{mol} / \mathrm{m}^{3}\right)$. The adsorption density can be used in calculating the average parking area $(A)$ a molecule occupies at the interface ( $\mathrm{m}^{2} /$ molecule) through $A=1 / \Gamma N_{\mathrm{av}}$ where $N_{\mathrm{av}}$ is the Avogadro's number. The parking area provides valuable information on the degree of packing and the orientation of the adsorbed molecules.

The adsorption densities calculated from the surface tension data in Fig. 3 using Eq. (1) are $3.86 \times 10^{-2}, 3.33 \times 10^{-2}$ and $2.97 \times 10^{-2} \mathrm{~mol} / \mathrm{cm}^{2}$ for the SDS, CTAB and TX-100, respectively. The literature values for the same surfactants are $4.76 \times 10^{-2}$, $3.10 \times 10^{-2}$ and $2.83 \times 10^{-2} \mathrm{~mol} / \mathrm{cm}^{2}$ [22-24]. The respective parking areas calculated from these densities are $0.43,0.49$ and $0.55 \mathrm{~nm}^{2}$ per molecule for these surfactants. The differences in the surface tension values of these surfactants at a fixed concentration can be attributed to the differences in surface activity. The significantly lower surface tensions obtained with TX-100 point to a higher surface activity most probably due to its larger molecular weight compared to the other two surfactants.

The surface tension measurements were repeated in the presence of a fixed amount of $\mathrm{MB}\left(10^{-5} \mathrm{M}\right)$. These results are presented together with the results presented in Fig. 3 for comparison purposes (Fig. 4). The figure also shows the surface tension as a function of MB concentration when there are no surfactants in the system as an inset on the top figure.

The figure clearly shows that interfacial energies do not change significantly for the CTAB and TX-100 surfactants when MB molecules are present. The surface tensions in the micelle range also remain unaffected by the presence of MB for these surfactants. The data indicate that the interaction of the cationic MB with the cationic CTAB and nonionic TX-100 surfactants is limited. However, the lower interfacial energies obtained with the anionic SDS when the MB is present show that the surface activity is higher at low concentrations of the surfactant where the monomers of SDS are present. The presence of MB does not seem to have an effect on the interfacial energies where SDS micelles form.

The tests presented in Fig. 4 are repeated for 1:1 SDS/MB molecular ratio and presented in Fig. 5. The data show that the effect of the presence of MB on the surface activity of the SDS is more 


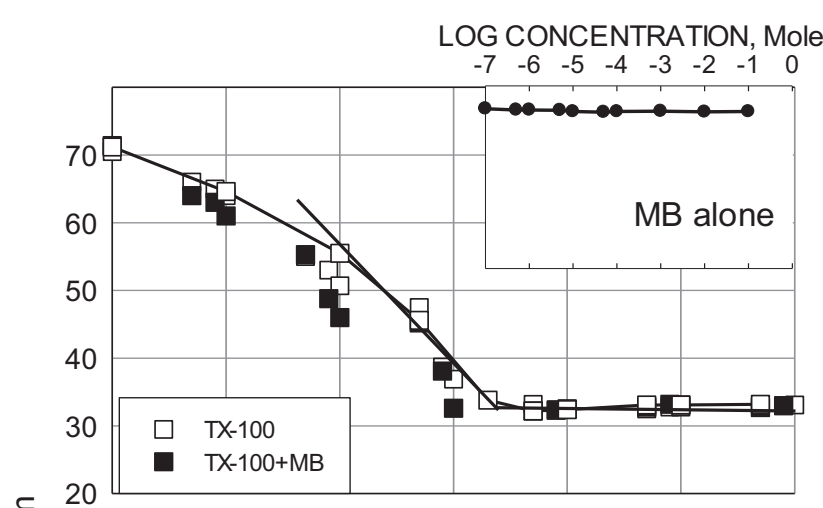

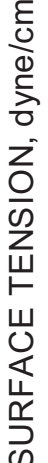

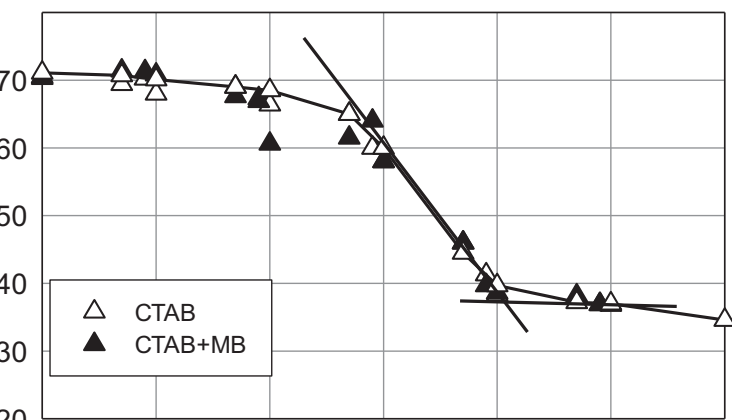
20

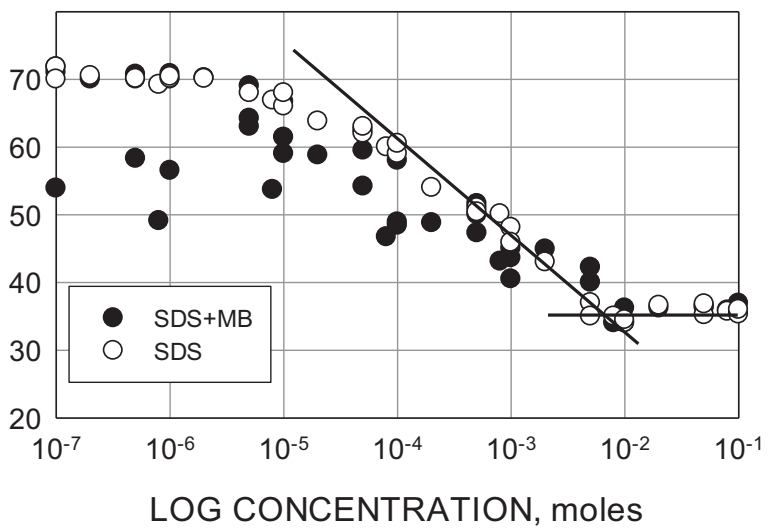

Fig. 4. Surface tensions of SDS, CTAB and TX-100 in the presence of $10^{-5} \mathrm{M} M B$ (2 ppm).

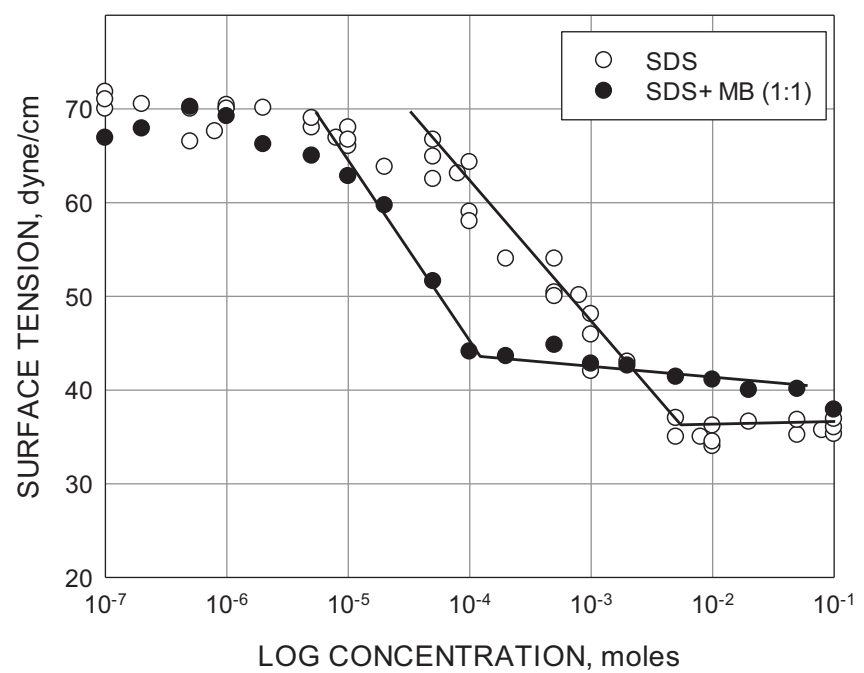

Fig. 5. Surface tension results of SDS in the presence of 1:1 SDS/MB molecular ratio. pronounced and extends to the micellar range, indicating a strong interaction between SDS and MB molecules. Fig. 5 also shows clearly that the presence of MB affect the CMC of the SDS significantly, more than an order of magnitude when MB concentration was relatively high (at a 1:1 ratio with the SDS). However, when the MB concentration is lower compared to the SDS, this effect becomes much smaller as can be seen from Fig. 4 .

\subsection{Charge and size of surfactant micelles in the absence and presence of $M B$}

The charge and size measurements were carried out to elucidate more clearly the interactions between the surfactant and MB molecules in the micellar range. The charge on the MB molecules was positive at the natural $\mathrm{pH}$ of 7.0 in the absence of any surfactants. The changes in the charging behavior of the surfactant micelles in the presence of MB are presented (Fig. 6). The figure shows that the changes were not significant at this concentration of $10^{-5} \mathrm{M} \mathrm{MB}$ ( $\left.\sim 2 \mathrm{ppm}\right)$. It was not possible to obtain charge data at higher concentrations of MB due to problems with the transparency of the solution to the laser beam of the charge measurement system. Similarly the changes in the size of micelles were also not significant at this concentration of $10^{-5} \mathrm{M} \mathrm{MB}(2 \mathrm{ppm})$ and it was not possible to obtain size data at higher concentrations of MB due to the similar problems.

\subsection{Contact angle of surfactant treated CNM filters in the absence and presence of $M B$}

Contact angle measurements were carried out with the CNM filter papers in various solution media: in plain water and in water containing only $\mathrm{MB}$ and in surfactant solutions in the presence and absence of MB to elucidate the interaction of the filter surface with the MB and surfactant molecules and with MB-surfactant aggregates. If present, the concentration of the $\mathrm{MB}$ was fixed at $10^{-5} \mathrm{M}$ in all tests. However, two different surfactant concentrations were tested: $10^{-5} \mathrm{M}$ to represent monomer range and $10^{-2} \mathrm{M}$ for micellar range.

The results of the contact angle studies are presented in Fig. 7a-c for the SDS, CTAB and TX-100 surfactants, separately. Note that the very first black bar on the left in each graph (no MB and no surfactant) corresponds to the contact angles measured for the filters in pure water. The gray bar adjacent to it is the contact angle value measured on the filter paper in water containing $10^{-5} \mathrm{M} \mathrm{MB}$ in the absence of any surfactant molecules.

It can be seen from the figures that the anionic filter surface is quite hydrophilic when measured in pure water. However, the filter surface becomes significantly hydrophobic in $10^{-5} \mathrm{M}$ MB solutions (no surfactant); a clear indication that the cationic MB molecules interact strongly with the negatively charged filter surface.

In the absence of $\mathrm{MB}$ in solution, the filter surface becomes quite hydrophobic at a surfactant concentration of $10^{-5} \mathrm{M}$ where all the surfactant molecules in solution are expected to be in the form of monomers (black bars at $10^{-5}$ and $10^{-2} \mathrm{M}$ surfactant concentrations). The degree of hydrophobicity imparted by the surfactant monomers increases with surfactant type, in the order SDS $\left(25^{\circ}\right)$, TX-100 $\left(45^{\circ}\right)$ and $\mathrm{CTAB}\left(65^{\circ}\right)$. The fact that the negatively filter surface is least affected by the anionic SDS and most affected by the cationic CTAB is another indication of the electrostatic interactions between the membrane surface and surfactant molecules. However, increased hydrophobicity by the non-ionic TX-100 suggests that hydrophobic interactions may also be playing a role.

At the surfactant concentration of $10^{-2} \mathrm{M}$ where the surfactant molecules form micelles (again in the absence of MB in solution), the contact angles on the membrane filter is very close to the values obtained in pure water. It seems that the presence of surfactants 

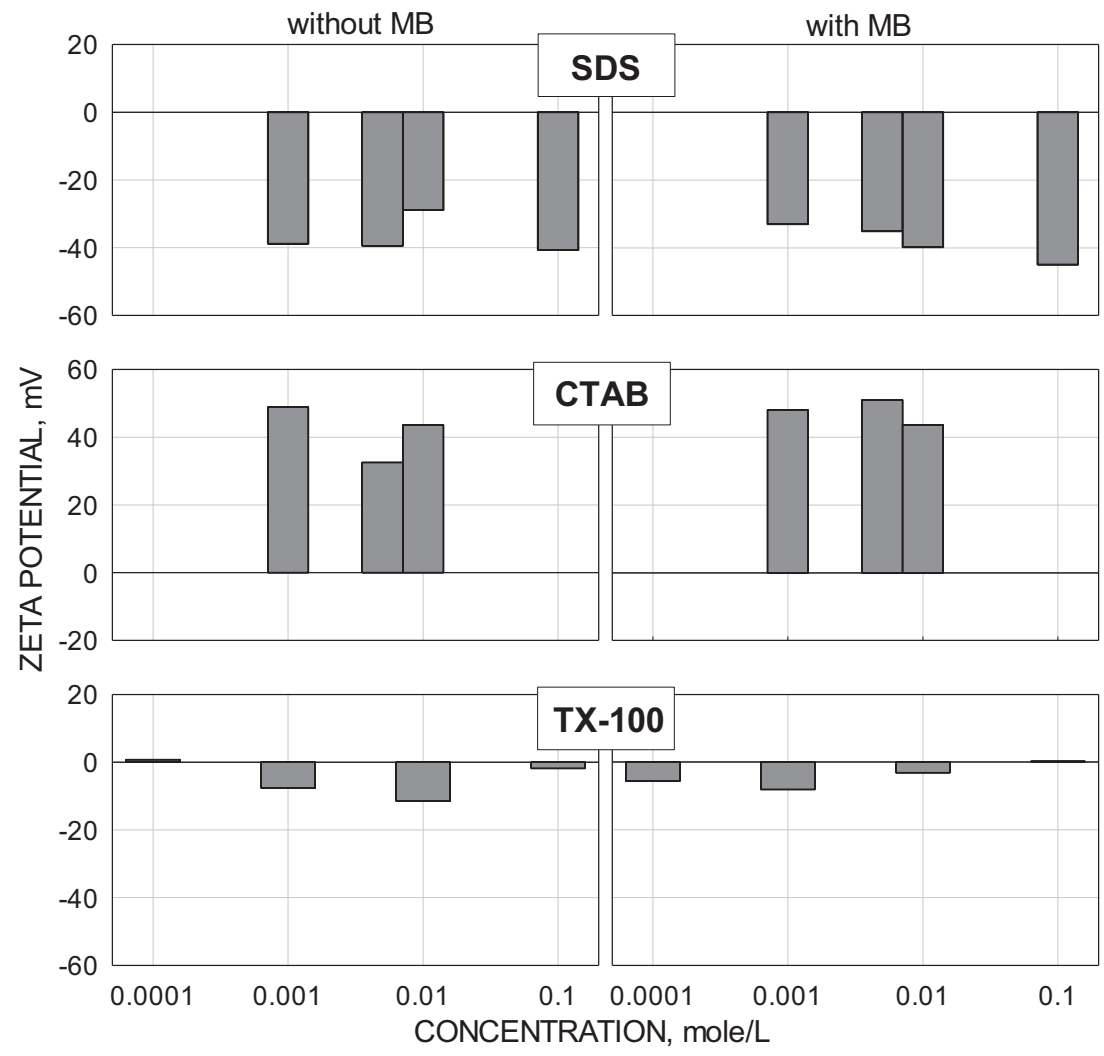

Fig. 6. Charge of SDS, CTAB and TX-100 micelles in the absence and presence of $10^{-5} \mathrm{M} \mathrm{MB}(2 \mathrm{ppm})$.
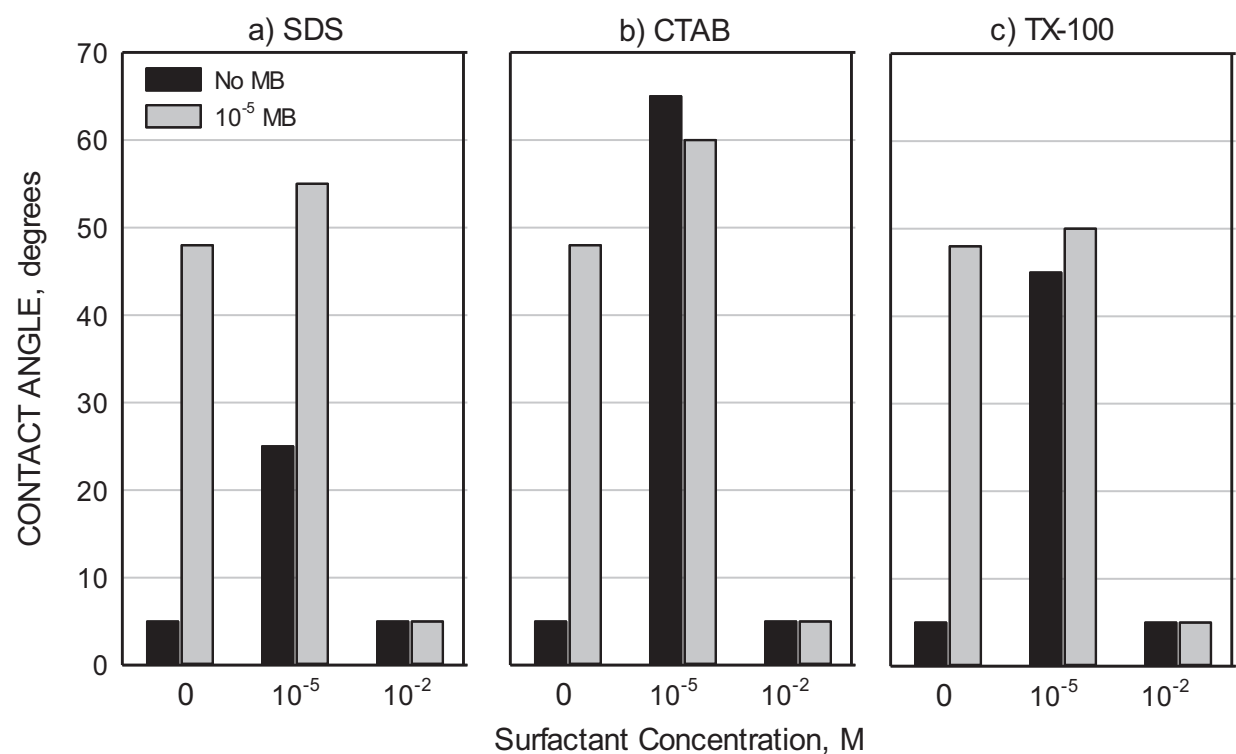

Fig. 7. Contact angle values on cellulose nitrate membrane (CNM) filters in water, methylene blue and methylene blue-surfactant solutions.

has no effect on the contact angle if the surfactant molecules are in micelle form. Since the anionic SDS micelles are not expected to adsorb on the negatively charged surface, this result is not surprising. As for the TX-100 micelles, it seems that hydrophobic interactions are also shielded in micelle form. The low contact angles observed for CTAB is most probably due to the hydrophilic nature of the CTAB micelles adsorbed on the surface.

When surfactants are added into solutions containing $10^{-5} \mathrm{M}$ $\mathrm{MB}$ (gray bars at $10^{-5}$ and $10^{-2} \mathrm{M}$ surfactant concentrations), hydrophobicity of the membrane surface does not change signif- icantly for CTAB and TX-100 compared to the case with no MB. However, hydrophobicity of the membrane surface increases significantly when $\mathrm{MB}$ is present in the solution together with the SDS. This increase in the contact angle when SDS co-exists with MB in solution (which surpasses the contact angles obtained with MB alone) indicates a synergistic interaction between the SDS and MB molecules on the membrane surface.

On the other hand, the contact angles obtained on the membrane surface when surfactants micelles in solution co-exist with $\mathrm{MB}$ are very low; very similar to that observed in the case if there 


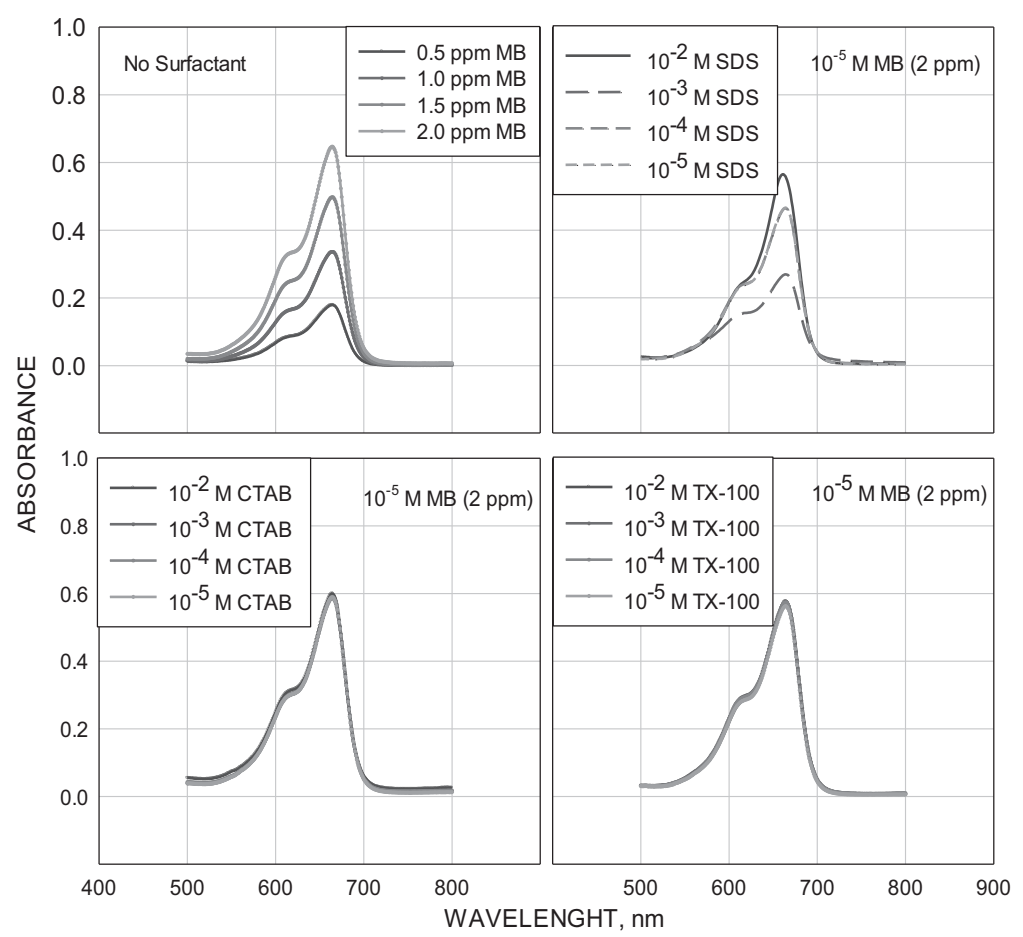

Fig. 8. UV-absorbance of $M B$ in the absence and the presence of SDS/CTAB/TX-100 of surfactants.

were no MB in the system. This result is rather interesting since it points out the probability that MB molecules, which in their free form make the surface hydrophobic may not be free to adsorb to the surface and may be locked in the structure of the micelles.

These results demonstrate that the interactions of the surfactant molecules or the MB-surfactant aggregates with the filter surface are complex. The results clearly show that filtration efficiency should be expected to depend not only on the interactions between the $\mathrm{MB}$ and surfactant molecules, but also the interactions between the filter surface and the dissolved species which may be present in the system. All these will be discussed in the following paragraphs after the presentation of filtration results.

\subsection{UV absorbance of SDS, CTAB, TX-100 surfactant solutions}

Prior to ultrafiltration experiments, UV-analyses were conducted to understand the effect of surfactants on the UVabsorbance of MB (Figs. 8 and 9). The top left graph in Fig. 8 gives the raw absorbance values as a function of wavelength for different MB concentrations. The other three graphs in the figure give the raw absorbance values for surfactant solutions containing $10^{-5} \mathrm{M}$ ( $\sim 2 \mathrm{ppm}) \mathrm{MB}$ (top right: SDS + MB; bottom left: $\mathrm{CTAB}+\mathrm{MB}$; bottom right: $\mathrm{TX}-100+\mathrm{MB}$ ). The $\mathrm{MB}$ graph shows that the maximum absorbance at $664 \mathrm{~nm}$ corresponds well with the MB concentration in solution. The maximum absorbance value for $2 \mathrm{ppm}$ is around 0.64 . Adding varying amounts of CTAB and TX-100 to a 2 ppm MB solution does not seem to have a significant effect in the measured absorbance values (see also Fig. 9).

However, this is not the case for the negatively charged SDS. The absorbance values varied at different additions of the surfactant to the $2 \mathrm{ppm} \mathrm{MB}\left(\sim 10^{-5} \mathrm{M} \mathrm{MB}\right)$ solution as can be seen in Fig. 8 . The maximum absorbance values measured at different strength SDS and MB solutions show this behavior more clearly (Fig. 9). It can be seen that the maximum absorbance value is changing, especially around $10^{-3} \mathrm{M}$ concentration of the surfactant which is very close to the CMC value $\left(8.0 \times 10^{-3} \mathrm{M}\right)$. This is another indication of the interaction between the anionic SDS and the cationic dye. However, since this effect is insignificant at the MB concentration $\left(10^{-5} \mathrm{M}\right.$ $\mathrm{MB}$ ) used in this study, UV-absorbance method was employed to determine the MB concentrations in the presence of SDS at all the concentrations.

\subsection{Filtration experiments: removal of $\mathrm{mb}$ in the presence of $S D S$, TX-100 and CTAB}

In this part of the study, filtration experiments were carried out with CNM filters using MB solutions at different surfactant concentrations in order to see the effect of surfactants in the filtration behavior (Fig. 10).

Fig. 10a gives the filtration efficiencies (\% removal) of pure $\mathrm{MB}$ solutions at $0.5-2.0 \mathrm{ppm}$ concentration range using filters of $0.45 \mu \mathrm{m}$ pore size. It is important to note that the filters were able to capture around $80 \%$ of the MB in solution in a single pass in this concentration range. This result is interesting in that the positively charged MB molecules seem to easily adsorb on the negatively charged filter surface most probably through electrostatic attraction in the absence of surfactants. Hence, contamination and fouling of the filter surface by the contaminant should be taken into account seriously.

It was observed that removal of MB is affected quite significantly by the presence of surfactants (Fig. 10b-d). The effect is a strong function of the type and concentration of the surfactant present in the system. In all cases, MB removal decreased progressively to very low values with increasing surfactant concentration. Another interesting observation is the fact that the decrease in the removal efficiency for SDS and TX-100 is precipitous and coincides with the CMC of these surfactants. However, for the cationic CTAB, the decrease starts from low surfactant concentrations and proceeds more slowly as surfactant concentration increases.

Based on the results of filtration experiments along with the results obtained from surface energy, contact angle and charge measurements the following mechanism is suggested for the observed behavior: 


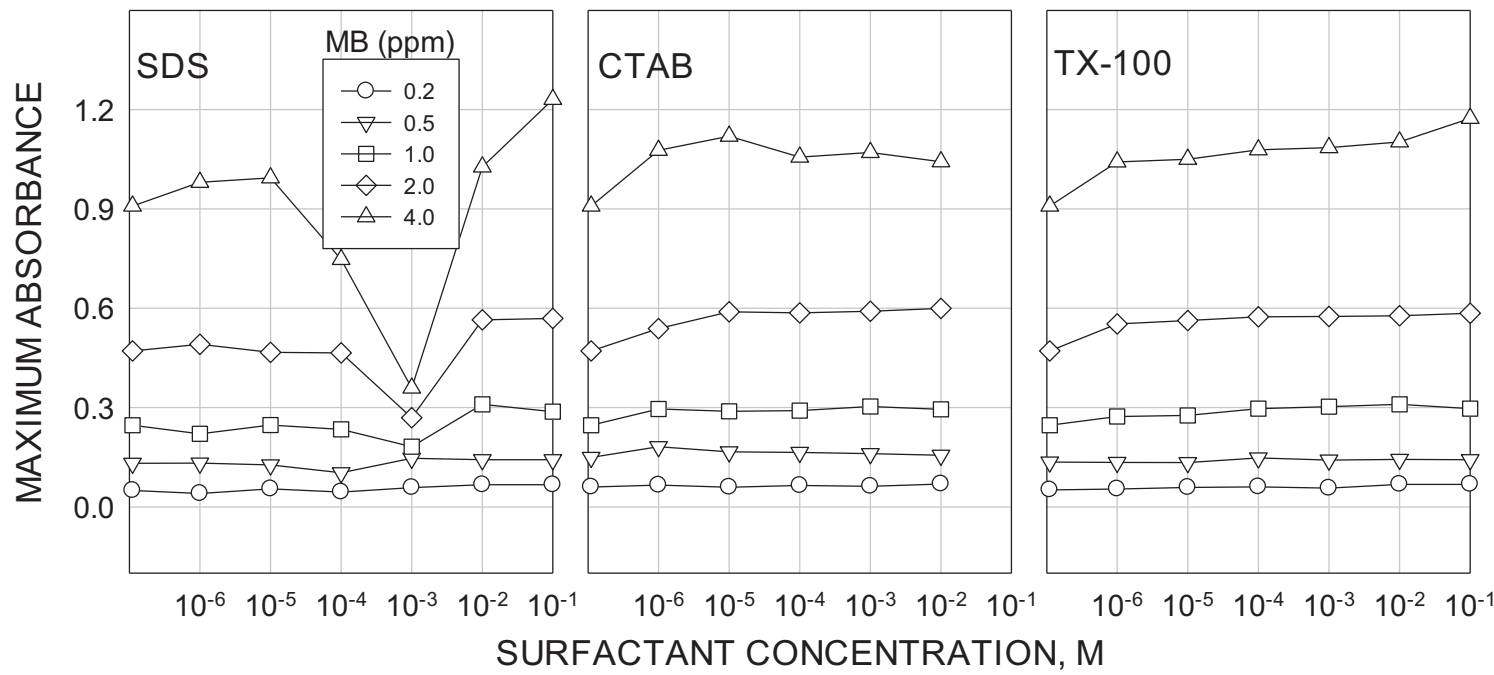

Fig. 9. Maximum UV-absorbance of MB in the presence of SDS/CTAB/TX-100 surfactants.
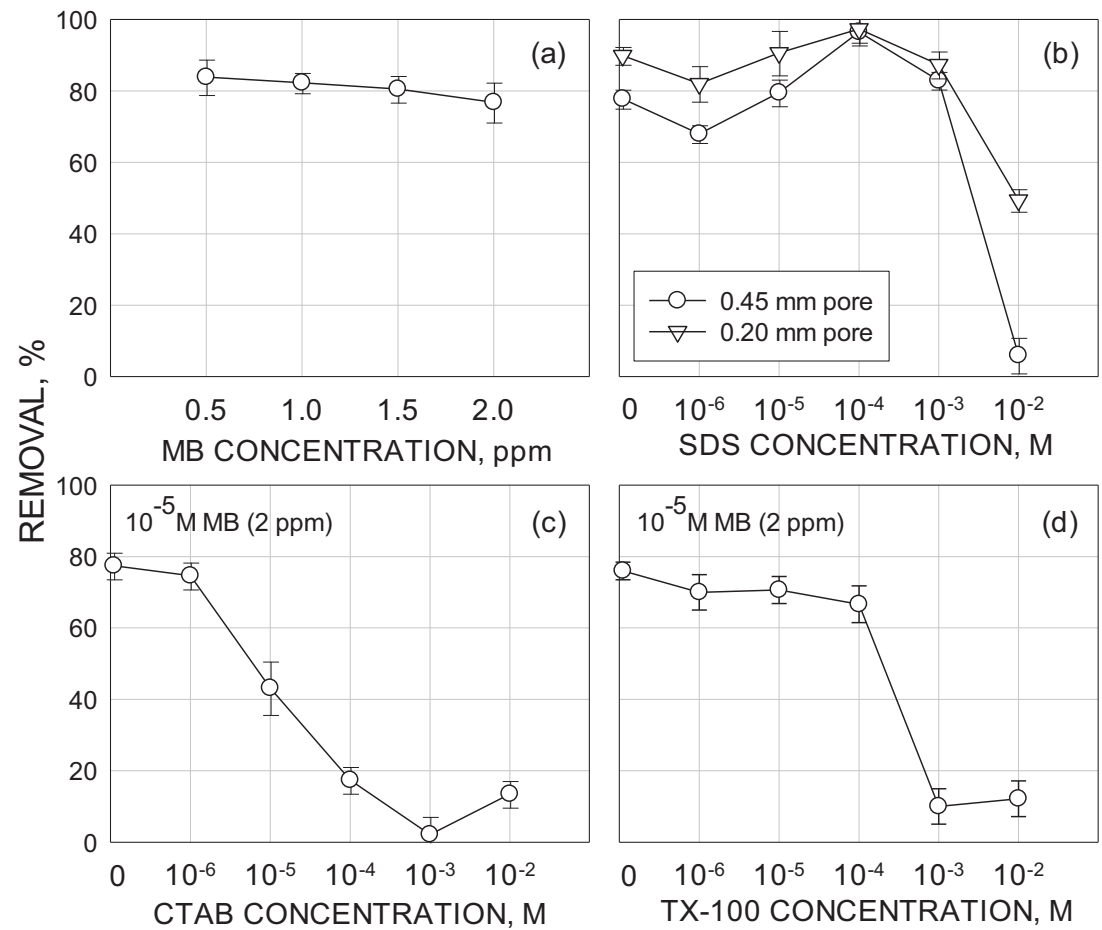

SDS CONCENTRATION, M

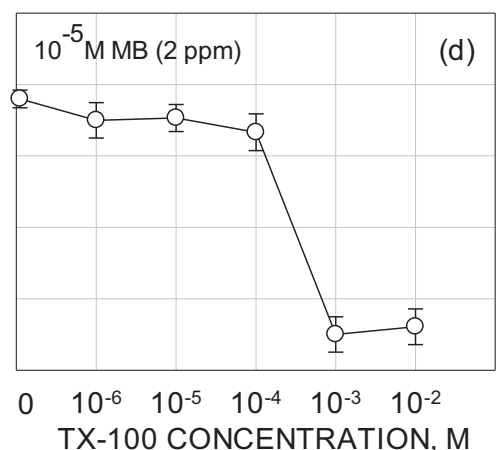

Fig. 10. MB removal by filtration in the absence and presence of surfactants, SDS, CTAB, TX-100.

In the case of anionic SDS, cationic MB is expected to interact electrostatically with the monomers of this surfactant to form meshed MB-SDS aggregates. The observed increase in the removal of MB compared to that observed with the MB alone (Fig. 10a) at low SDS concentrations can be due to the inability of these MBSDS aggregates to pass through the pores of the filter. The higher removal efficiencies observed with the smaller $0.20 \mu \mathrm{m}$ pore filter paper supports this hypothesis. These results are also in accordance with the contact angle results presented previously in Fig. 7.

After CMC, MB molecules are most probably included in the structure of the SDS micelles. The fact that the removal efficiency decreases suddenly after the CMC could be due to the inability of the filter to retain these micelles which are smaller than the pore size of the filter. The larger removal efficiency with the $0.20 \mu \mathrm{m}$ pore size filter (50\%) compared to that observed with the $0.45 \mu \mathrm{m}$ pore size filter (5\%) strongly supports this theory.

In the case of $C T A B$, both the CTAB and $\mathrm{MB}$ molecules are expected to interact with the filter surface electrostatically. The contact angle results given in Fig. 7 is a proof of this interaction. Hence, there will be a competition between the MB and CTAB monomers for the filter surface. Since cationic CTAB cannot interact with the cationic MB, structures similar to the meshed MB-SDS aggregates cannot be expected for this surfactant, precluding any physical retention of $\mathrm{MB}$ on the filter surface. As a result of the competition stated above, the removal efficiency of the MB molecules should decrease as CTAB concentration increases as observed in Fig. 10c. In the micelle range, similar to the SDS case, compact $\mathrm{CTAB}$ micelles which include the MB molecules pass through the $0.45 \mu \mathrm{m}$ pore size filter relatively unhindered. The slight increase 
in the removal efficiency after the CMC may be due to some degree of adsorption of the cationic micelles on the negative filter surface.

In the case of TX-100, the contact angle results given in Fig. 7 show that TX-100 adsorb at the filter surface. However, the negatively charged filter surface should favor MB adsorption, especially at low concentration of the surfactant. This is shown by the removal efficiencies in Fig. 10d, which are very similar to those observed for $\mathrm{MB}$ alone in Fig. 10a. As the concentration of surfactant increases, more TX-100 molecules can be expected on the filter surface, hampering $\mathrm{MB}$ adsorption to a degree, hence the slight decrease observed in the removal efficiency. After CMC, on the other hand the removal of $\mathrm{MB}$ decreases due to similar reasons explained for SDS.

\section{Conclusions}

In this study, a widely encountered LMWC, methylene blue, was chosen as the model contaminant. Anionic (sodium dodecyl sulfate, SDS), cationic (hexadecyltrimethyl-ammonium bromide, CTAB) and non-ionic (ethoxylated octylphenol, TX-100) surfactants were used as model surface active agents to investigate their interactions with methylene blue. The MB-surfactant systems were characterized through surface tension, charge and contact angle measurements in order to elucidate the forms of surfactant and MB-surfactant molecules in aqueous solutions as a function of concentration. These studies also provided information on the critical concentrations where the micelles form in the absence and presence of MB.

The simulated contaminated waters (MB-surfactant solutions) were subjected to filtration tests to correlate the findings of the characterization studies with filtration efficiencies. Ultraviolet-Vis spectroscopy (UV) was used to determine the MB concentrations in these studies. Based on the findings of this study the following conclusions could be made:

- There is an electrostatic interaction between the filter paper and MB which leads to significant removal of this contaminant due to this effect alone in one pass. Hence, contamination and fouling of the filter surface by the contaminant should be taken into account seriously.

- Presence of surfactant changes the filtration behavior drastically. In the monomer range, removal efficiency decreases with increasing surfactant concentration in general. The magnitude and rate of this decrease depend strongly on the charge of the surfactant. For example, the cationic CTAB competes for the membrane surface with the cationic $\mathrm{MB}$ and decreases removal to a great degree as its concentration increases. This shows that the interactions between the filter surface and the surfactant molecules are as important as those between the filter surface and the contaminant.

- Interactions between the contaminant and the surfactant molecules are also important since they determine the form of the contaminant in the system, hence the filtration response. For example, the interaction of the anionic SDS with MB seems to create meshed SDS-MB aggregated which favors removal efficiency.

- In the micellar range, the efficiency of removal is low for all surfactants most probably due to the formation of compact surfactant micelles, which envelope MB in their structure, which cannot be retained by the pores of the filters used in this study.

\section{References}

[1] Y.K. Choi, S.B. Lee, D.J. Lee, Y. Ishigami, T. Kajiuchi, Micellar enhanced ultrafiltration using PEO-PPO-PEO block copolymers, J. Membr. Sci. 148 (1998) 185-194.

[2] P.N. Hurter, T.A. Hatton, Solubilization of polycyclic aromatic hydrocarbons by poly(ethylene oxide-propylene oxide) block copolymer micelles: effects of polymer structure, Langmuir 8 (1992) 1291-1299.

[3] M.T. Yagub, T.K. Sen, S. Afroze, H.M. Ang, Dye and its removal from aqueous solution by adsorption: a review, Adv. Colloid Interface Sci. 209 (2014) $172-182$.

[4] A.F. Hassan, A.M. Abdel-Mohsen, M.M.G. Fouda, Comparative study of calcium alginate, activated carbon, and their composite beads on methylene blue adsorption, Carbohydr. Polym. 102 (2014) 192-198.

[5] A. Gürses, A. Hassani, M. Kıranşan, Ö. Açışlı, S. Karaca, Removal of methylene blue from aqueous solution using by untreated lignite as potential low-cost adsorbent: kinetic, thermodynamic and equilibrium approach, J. Water Process Eng. 2 (2014) 10-21.

[6] I.A. Aguayo-Villarreal, V. Hernández-Montoya, N.A. Rangel-Vázquez, M.A. Montes-Morán, Determination of QSAR properties of textile dyes and their adsorption on novel carbonaceous adsorbents, J. Mol. Liq. 196 (2014) 326-333.

[7] S.M. Doke, G.D. Yadav, Novelties of combustion synthesized titania ultrafiltration membrane in efficient removal of methylene blue dye from aqueous effluent, Chemosphere 117 (2014) 760-765.

[8] S. Dawood, K.T. Sen, Review on dye removal from its aqueous solution into alternative cost effective and non-conventional adsorbents, J. Chem. Process Eng. 1 (2014) 1-11.

[9] J.H. Huang, Y.L. Xiong, G.M. Zeng, S.H. Guo, G.X. Xie, D.M. Zhang, X.J. Tang, Z.F. Liu, Separation of phenol from various micellar solutions using MEUF, Sep. Purif. Technol. 98 (2012) 1-6.

[10] M.A. Khosa, S.S. Shah, M.F. Nazar, Application of micellar enhanced ultrafiltration for the removal of methylene blue from aqueous solution, J. Dispersion Sci. Technol. 32 (2) (2011) 260-264.

[11] M.K. Purkait, S. DasGupta, S. De, Micellar enhanced ultrafiltration of eosin dye using hexadecyl pyridinium chloride, J. Hazard. Mater. 136 (2006) 972-977.

[12] K. Baek, H.-H. Lee, J.-W. Yang, Micellar-enhanced ultrafiltration for simultaneous removal of ferricyanide and nitrate, Desalination 158 (2003) $157-166$.

[13] N. Zaghbani, A. Hafiane, M. Dhahbi, Separation of methylene blue from aqueous solution by micellar enhanced ultrafiltration, Sep. Purif. Technol. 55 (2007) 117-124

[14] N. Zaghbani, A. Hafiane, M. Dhahbi, Removal of Eriochrome Blue Black R from wastewater using micellar-enhanced ultrafiltration, J. Hazard. Mater. 168 (2009) 1417-1421

[15] M. Ruzitah, A. Sharifah, An overview of micellar-enhanced ultrafiltration in wastewater treatment process, Int. Conf. on Environment and Industrial Innovation, IPCBEE 12 (2011) 167-172.

[16] J.-H. Huang, C.-F. Zhou, G.-M. Zeng, X. Li, H.-J. Huang, J. Niu, F. Li, L.-J. Shi, S.-B. He, Studies on the solubilization of aqueous methylene blue in surfactant using MEUF, Sep. Purif. Technol. 98 (2012) 497-502.

[17] K. Singh, N. Dharaiya, D.G. Marangoni, P. Bahadur, Dissimilar effects of solubilized $p$-toluidine on the shape of micelles of differently charged surfactants, Colloids Surf. A 436 (2013) 521-529.

[18] J.M. Walker, The Protein Protocols Handbook, 2nd edition, Springer Science \& Business Media, Humana Press, 2002, Section 40.3.2.

[19] P. Mukerjee, K.J. Mysels, Critical micelle concentrations of aqueous surfactant systems, J. Pharm. Sci. 61 (2) (1972) 319.

[20] K. Szymczyk, B. Jańczuk, The adsorption at solution-air interface and volumetric properties of mixtures of cationic and nonionic surfactants, Colloids Surf. A 293 (2007) 39-50.

[21] L. Tofani, A. Feis, R.E. Snoke, D. Berti, P. Baglioni, G. Smulevich, Spectroscopic and interfacial properties of myoglobin/surfactant complexes, Biophys. J. 87 (2004) 1186-1195.

[22] A. Zdziennicka, The adsorption properties of short chain alcohols and Triton X-100 mixtures at the water-air interface, J. Colloid Interface Sci. 335 (2009) $175-182$.

[23] T.D. Gurkova, D.T. Dimitrovaa, K.G. Marinovaa, C. Bilke-Crauseb, C. Gerberb, I.B. Ivanova, Ionic surfactants on fluid interfaces: determination of the adsorption; role of the salt and the type of the hydrophobic phase, Colloids Surf. A 261 (2005) 29-38.

[24] K. Szymczyk, B. Janczuk, The adsorption at solution-air interface and volumetric propertiesof mixtures of cationic and nonionic surfactants, Colloids Surf. A 293 (2007) 39-50. 